\title{
ON THE POLARIZATION AND ANISOTROPY \\ OF SOLAR X-RADIATION DURING FLARES
}

\author{
G. ELWERT and E. HAUG
}

Lehrstuhl für Theoretische Astrophysik der Universität, Tübingen, D.B.R.

From the spectral intensity distribution of solar X-radiation one can conclude that there is a non-thermal contribution, which is effective during the initial phase of a flare. It is reasonable to suppose that the electrons producing the non-thermal radiation have a non-isotropic velocity distribution. Indeed, one should assume that the electrons, during their acceleration, obtain a preferred direction and that only afterwards does their velocity distribution become Maxwellian by means of collisions. Hence the short-wavelength continuous X-radiation in the initial phase of a flare, consisting mainly of bremsstrahlung, should be polarized and should have a non-isotropic angular distribution.

In papers presented to the IAU-Symposia in Budapest 1967 (Elwert, 1968) and in Leningrad last May, the polarization of X-radiation from electrons of a few $\mathrm{keV}$ accelerated in solar flares has been calculated using energy distributions based on the observations of Pounds et al. obtained with the satellites Ariel I and OSO-4.

With the aid of these measurements the electron spectra can be derived up to an energy of 4 to $10 \mathrm{keV}$. Using these spectra the polarization of the $\mathrm{X}$-radiation was calculated by means of the nonrelativistic formula for the differential bremsstrahlung cross-section. For a vanishing magnetic field, the polarization $P$ for a photon energy $h v=3 \mathrm{keV}$ is given by the lower curve in Figure $1, \vartheta$ being the angle between the electron velocity and the line of sight. Taking into account a magnetic field, the polarization curves are represented for various pitch angles $\alpha, \vartheta$ being now the angle between the direction of the magnetic field and the line of sight.

Considering the case where the directions of the electron velocities are distributed according to a function $F_{n}(\alpha)=\sin ^{n} \alpha$ as used by Korchak, the polarization curves have a single sign owing to the predominance of the large angles $\alpha$ near $90^{\circ}$. The degree of polarization is reduced to $30 \%$ at most (see Figure 2).

The measurement of polarization provides the possibility of testing various flare models. According to the model of De Jager and Kundu, the electrons move mainly radially. On the other hand Takakura and Kai proposed in their model, that the electrons move in the direction of the magnetic field of a bipolar sunspot, which is mainly horizontal at higher altitudes in the solar atmosphere. With the model of De Jager and Kundu, the degree of polarization is equal to zero if a flare occurs at the center of the Sun's disk, whereas it is highest at the solar limb. A horizontal movement results in a maximum polarization $P$ in the center, whereas $P$ vanishes at the limb of the Sun if the magnetic field is in the equatorial direction.

Recently Mandelshtam et al. (1970) have observed that the short-wavelength 


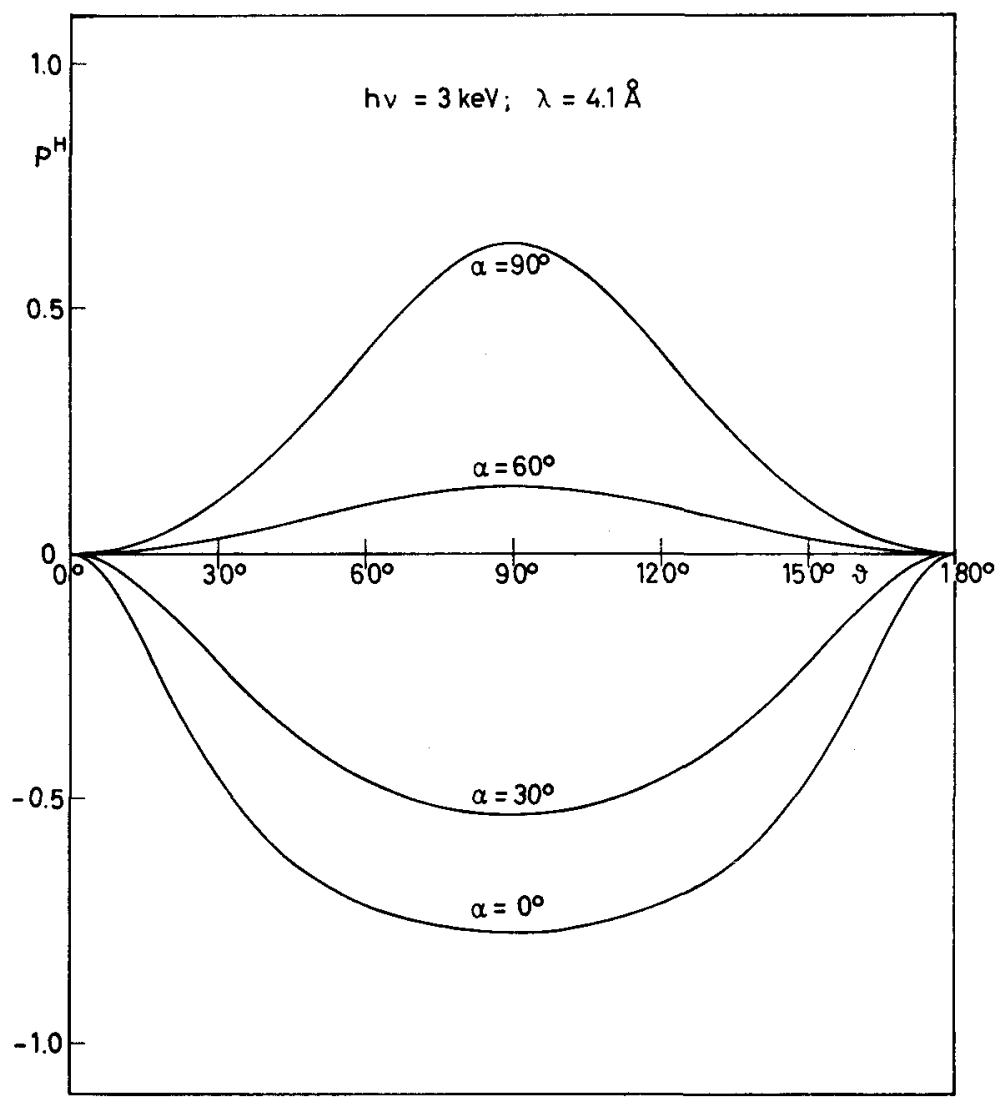

Fig. 1. The polarization at $4.1 \AA$ as a function of $\alpha$ and $\vartheta$ the pitch angle and the angle between the magnetic field and line of sight.

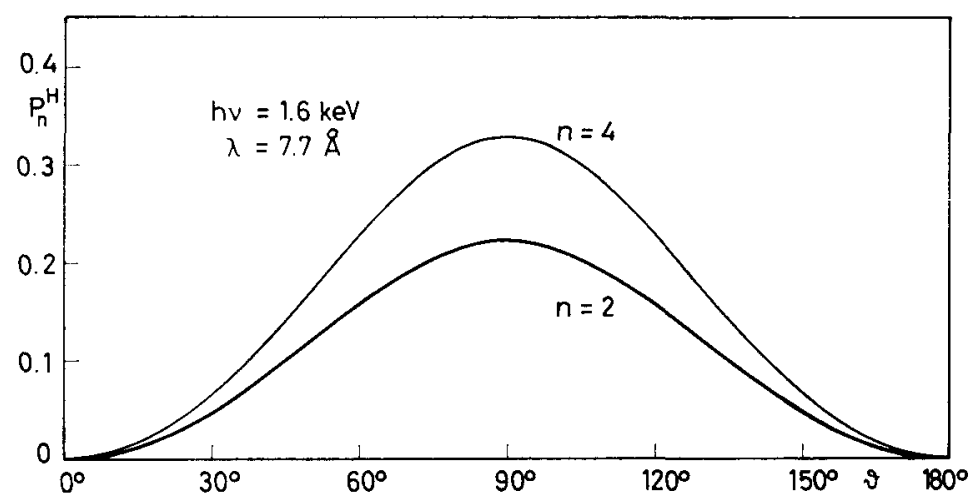

Fig. 2. The polarization as a function of $\vartheta$ for electron velocities distributed according to $F_{n}(\alpha)=\sin ^{n} \alpha$. 


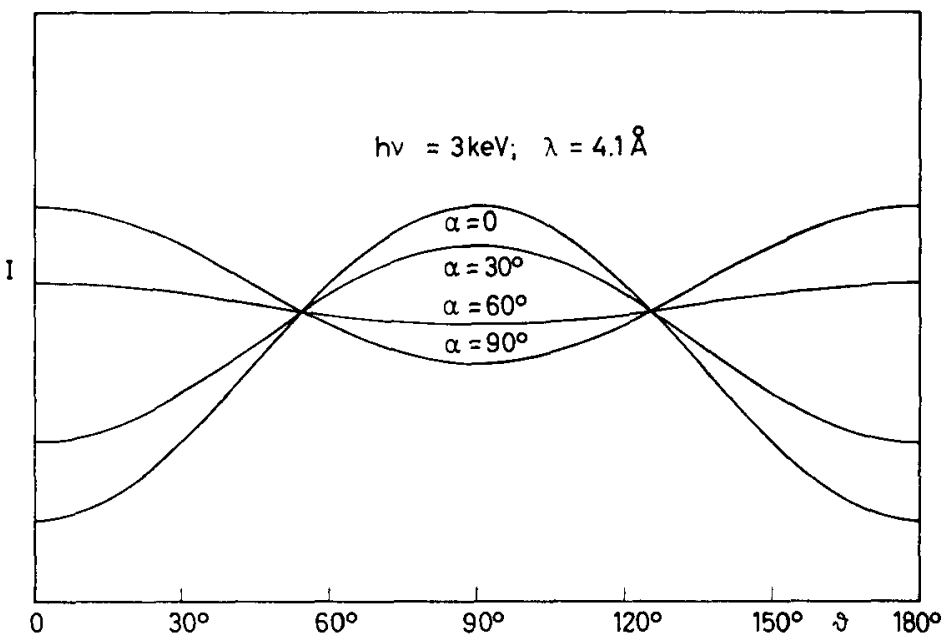

Fig. 3. The angular distribution of the intensity of X-radiation as a function of $\alpha$ and $\vartheta$.

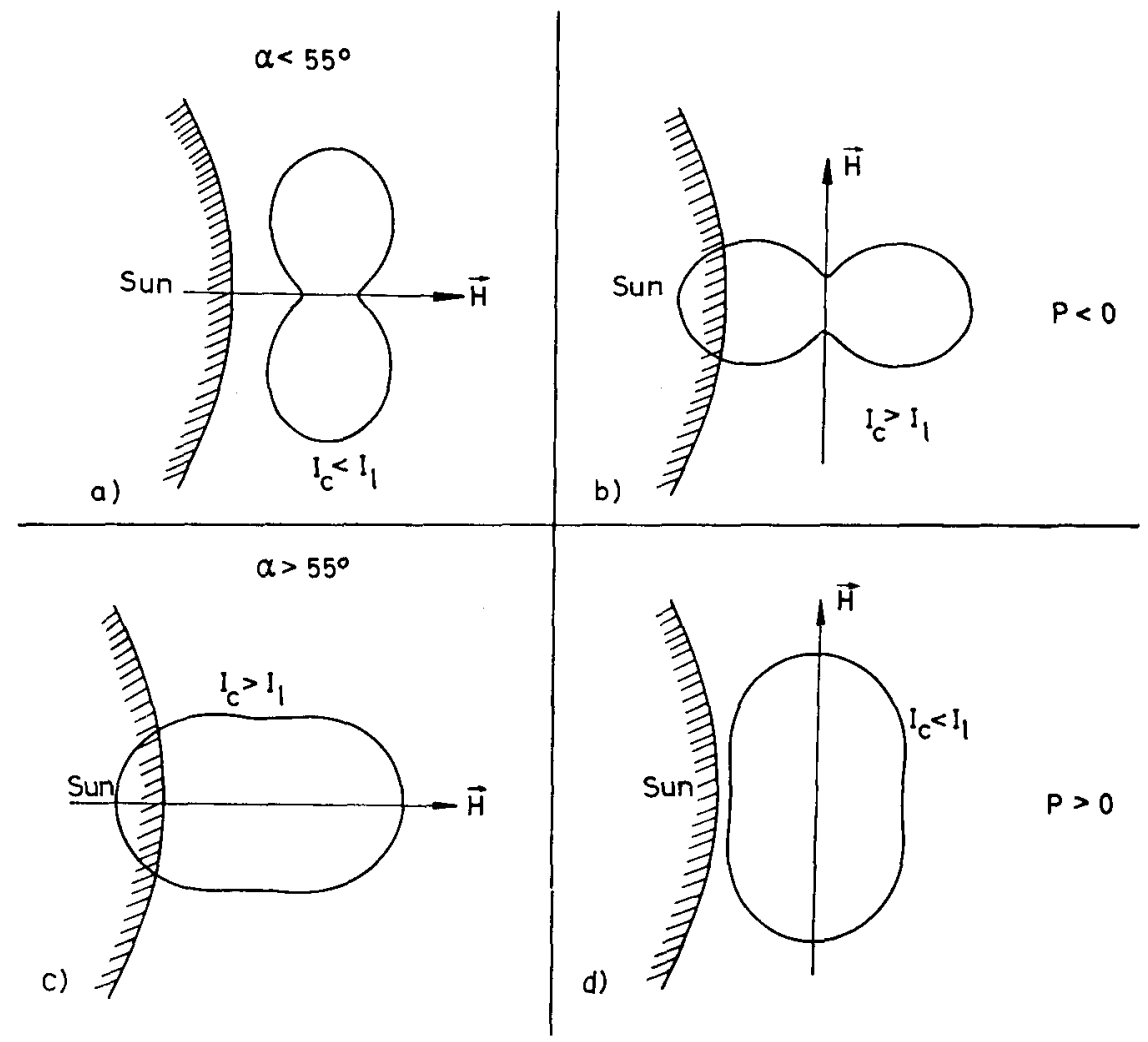

Fig. 4. Polar diagrams of the intensity distributions as a function of $\alpha$. 
solar X-rays are indeed polarized during flares. They recorded three small X-ray flares in October last year and obtained at $0.8 \AA$ a polarization of approximately $40 \%$.

This fact confirms that the electrons must have a preferred direction during the initial phase of flare. For this reason it might be interesting to consider the angular distribution of this X-radiation, for example for a photon energy of $3 \mathrm{keV}$. The results of the calculation are represented in Figure 3. In case of vanishing magnetic field or in case of the pitch angle $\alpha=0$, the intensity is most anisotropic with a maximum for $\vartheta=90^{\circ}$.

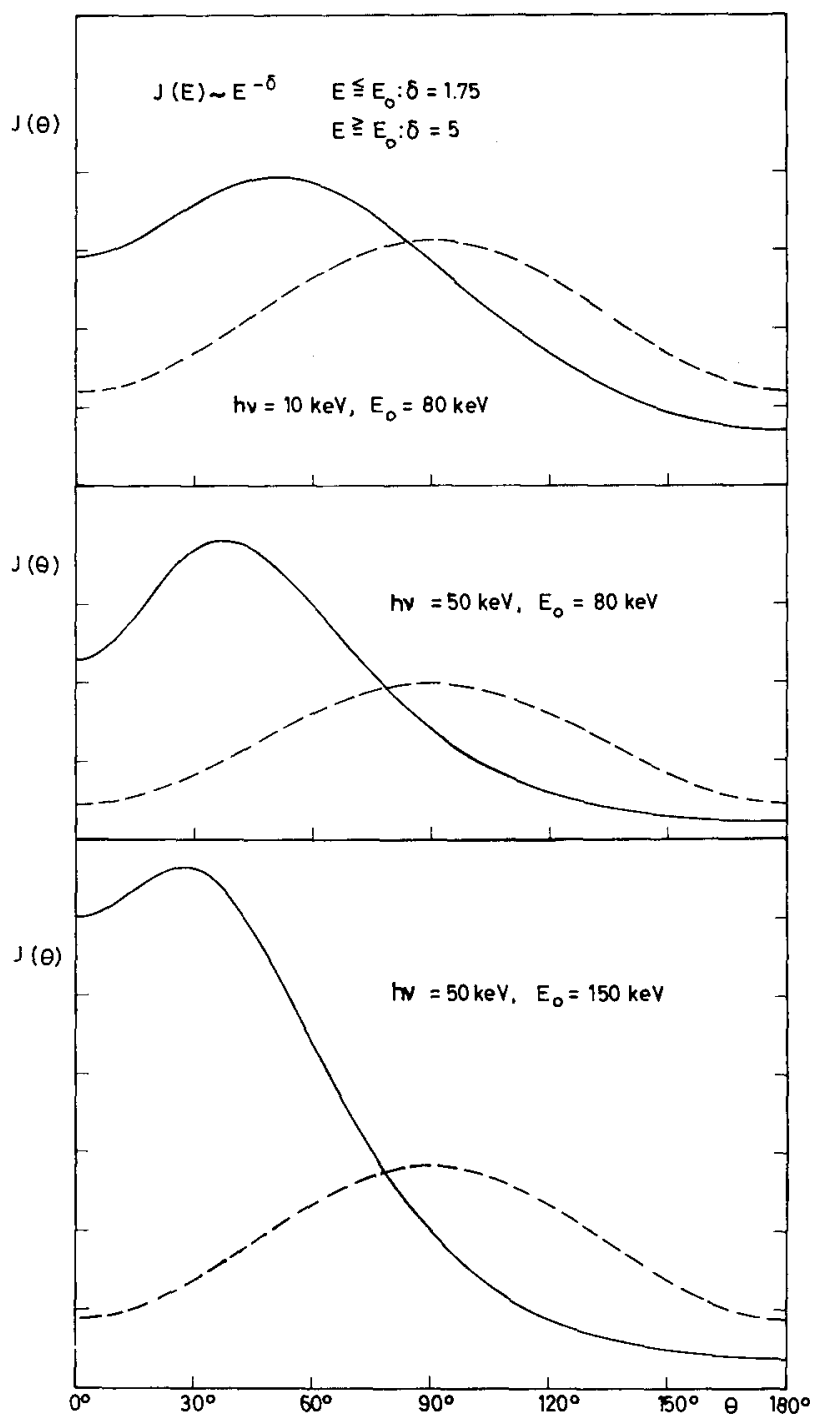

Fig. 5. The angular distribution for high photon energies for electron moving parallel to the magnetic field. 
Polar diagrams of the intensity distributions with the parameter $\alpha$ are represented in Figure 4. They give the intensities for radial and tangential magnetic fields in the equatorial plane. The spatial distributions are obtained by rotating these curves around the direction of the magnetic field. They have the appearance of dumb-bells for $\alpha<55^{\circ}$ and of tubes for $\alpha>55^{\circ}$, as shown in the lower and upper part of the figure, respectively. For $\alpha=55^{\circ}$ the intensity is isotropic. The curves represented correspond to $\alpha=0$ and $\alpha=90^{\circ}$. For a flare observed at the limb of the Sun, the line of sight is vertical, and for a flare at the center of the solar disk, the line of sight is horizontal. The diagrams on the left side refer to a radial magnetic field, the right side refers to a tangential field.

The angular distributions for higher photon energies, for instance for 10 and $50 \mathrm{keV}$ and for electrons moving parallel to the magnetic field are shown in Figure 5. In this case the nonrelativistic bremsstrahlung cross-section is no longer valid. Therefore the relativistic formula of Bethe and Heitler was used. From the observations of X-ray spectra performed by Kane and Anderson and by Frost a power law spectrum for the electron energies can be derived, and an abrupt increase in the slope of the electron spectra in the neighbourhood of an energy $E_{0}=100 \mathrm{keV}$ can be inferred. For energies $E \geqslant E_{0}$ one obtains the exponent $\delta=1.75$, whereas for $E \geqslant E_{0}$ the measurements are compatible with $\delta=5$. The angular distributions are dependent on the value of $E_{0}$ since the electrons with energies appreciably higher than the photon energy give an important contribution to the X-ray intensity. As a result the maximum of the angular distribution is peaked more and more in forward direction, for increasing values of the photon energy, $E_{0}$. For comparison, the distributions calculated with the nonrelativistic bremsstrahlung cross section are shown as broken curves, they are symmetric with respect to $90^{\circ}$. For $h v=10 \mathrm{keV}$, the difference is not yet pronounced, but it increases with increasing photon energy, especially for large values of $E_{0}$, i.e. if the change in the slope of the electron spectrum is at higher energy, as shown in the lower curves.

These angular distributions might be measured with the aid of satellites at great distances from the Earth. Then the possibility would arise to obtain new information about the physical processes occurring during flares.

\section{References}

Elwert, G.: 1968, in K. O. Kiepenheuer (ed.), 'Structure and Development of Solar Active Regions', IAU Symp. 35, 444.

Elwert, G. and Haug, E.: 1970, Solar Phys. 15, 234; generalization to relativistic electron energies to be published.

Tindo, I. P., Ivanov, V. D., Mandelshtam, S. L., and Shuryghin, A. I.: 1970, Solar Phys. 14, 204. 